\title{
In situ TEM Fracture Testing for Shallow Ion Irradiated Layers
}

\author{
Janelle P. Wharry ${ }^{1}$ and Kayla H. Yano ${ }^{2}$ \\ 1. School of Nuclear Engineering, Purdue University, West Lafayette, IN, USA \\ 2. Micron School of Materials Science \& Engineering, Boise State University, Boise, ID, USA
}

The objective of this work is to demonstrate in situ transmission electron microscopic (TEM) bend tests for evaluating the fracture toughness of materials. Conventional fracture toughness tests, such as Charpy $\mathrm{V}$-notch or compact tension (CT) configurations, require large specimen volumes having homogeneous properties and microstructures throughout that volume in order to obtain meaningful quantitative results [1]. But these test geometries are unfeasible for volume-limited materials such as thin films or ion implanted or irradiated layers, and for hazardous specimens that are difficult to handle in large quantities, such as radioactive materials. Hence, in situ TEM nanomechanical testing may offer a transformative method to evaluate the fracture properties of such materials. In situ TEM mechanical testing also offers the distinct advantage of enabling concurrent TEM-resolution imaging/video with quantitative mechanical testing of sub-micron-sized electron-transparent specimens, enabling direct linkage of plastic phenomena to mechanical behavior.

We focus this study on a model Fe-9Cr oxide dispersion strengthened (ODS) alloy (Fe-8.67Cr-1.95W$0.28 \mathrm{Y}-0.23 \mathrm{Ti}-0.14 \mathrm{C}-0.048 \mathrm{Si}-0.06 \mathrm{Ni}$, in wt.\%), subject to near-surface ion irradiation. Irradiations are carried out to 3 displacements per atom (dpa) at $500^{\circ} \mathrm{C}$ using $2 \mathrm{MeV}$ protons. The damage peak is calculated by SRIM-2013 [2] and is located $20 \mu \mathrm{m}$ below the surface; the 3 dpa dose level is attained at a depth $\sim 10 \mu \mathrm{m}$ below the surface, along the flattest region of the damage profile. Focused ion beam (FIB) machining is used to fabricate nanosized notched bend test specimens, the entire volume of which are located within the $3 \mathrm{dpa} \pm 0.5$ dpa irradiation dose level. Electron backscatter diffraction (EBSD) during FIB milling ensures that grain boundaries of interest are located below the FIB-machined notch in each specimen. Bend specimens are subsequently tested using a Hysitron PI95 fitted with a diamond flat punch. Two bend test geometries are investigated: cantilevers and 4-point bends.

Initial cantilever designs (Fig. 1a) range from 0.5-1 $\mu \mathrm{m}$ height, 3-8 $\mu \mathrm{m}$ length, and 75-200 nm thickness (electron transparency). Twelve and 13 cantilevers are tested from the unirradiated and irradiated ODS, respectively. None of the specimens experienced fracture. Some experienced extreme plastic flow (like superplasticity) in the region below the notch (Fig. 1b), while others experienced out-of-plane bending (Fig. 1c). These results led to the development of 4-point bend test geometries.

Four-point bend geometries secure the specimen on two ends, reducing the extent of out-of-plane bending. Further, the 4-point bend test geometry enables greater stress to be applied to the notch region, which may promote fracture. Typical dimensions of 4-point bend tests range over 0.5-1 $\mu \mathrm{m}$ height, 6-10 $\mu \mathrm{m}$ length, and 100-300 $\mathrm{nm}$ thickness (electron transparency). Eight unirradiated ODS specimens have thus far been tested to total loads $\sim 200 \mu \mathrm{N}$. One of the eight exhibited fracture at $202 \mu \mathrm{N}$; it has initial dimensions $750 \mathrm{~nm}$ height, $6.5 \mu \mathrm{m}$ length, and $300 \mathrm{~nm}$ thickness, with a $500 \mathrm{~nm}$ notch (Fig. 2). Based on a plane stress assumption [3], we can estimate from the specimen geometry a $\mathrm{K}_{\mathrm{IC}}$ value of 26.3 $\mathrm{MPa} \sqrt{\mathrm{m}}$, which is within the 12-30 $\mathrm{MPa} \sqrt{\mathrm{m}}$ known range of fracture toughness values from bulk Fe-9Cr ODS alloys [4]. 
Since traditional fracture mechanics break down at the nanomechanical length scale, future work involves utilizing extended finite element method (XFEM) modeling to more accurately represent the stress-strain conditions of the 4-point bend beams, in order to more meaningfully predict fracture toughness at these length scales. We will also conduct 4-point bend testing of the irradiated ODS [5].

\section{References:}

[1] M. A. Meyers and K. K. Chawla, Mechanical Behavior of Materials, 2nd ed. (Cambridge University Press) 2008.

[2] J. F. Ziegler, "The Stopping and Range of Ions in Matter (SRIM)," 2013.

[3] S. T. Pinho, P. Robinson, and L. Iannucci, Compos. Sci. Technol. 69, no. 7-8 (2009) p. 1303.

[4] B. Fournier et al, J. Nucl. Mater. 430 (2012) p. 142.

[5] We acknowledge Nuclear Regulatory Commission Grant NRC-HQ-84-14-G-0056, US DOE Nuclear Science User Facilities 15-569, the Idaho National Laboratory LDRD Program, the Micron Foundation, and the Microscopy \& Characterization Suite at CAES.
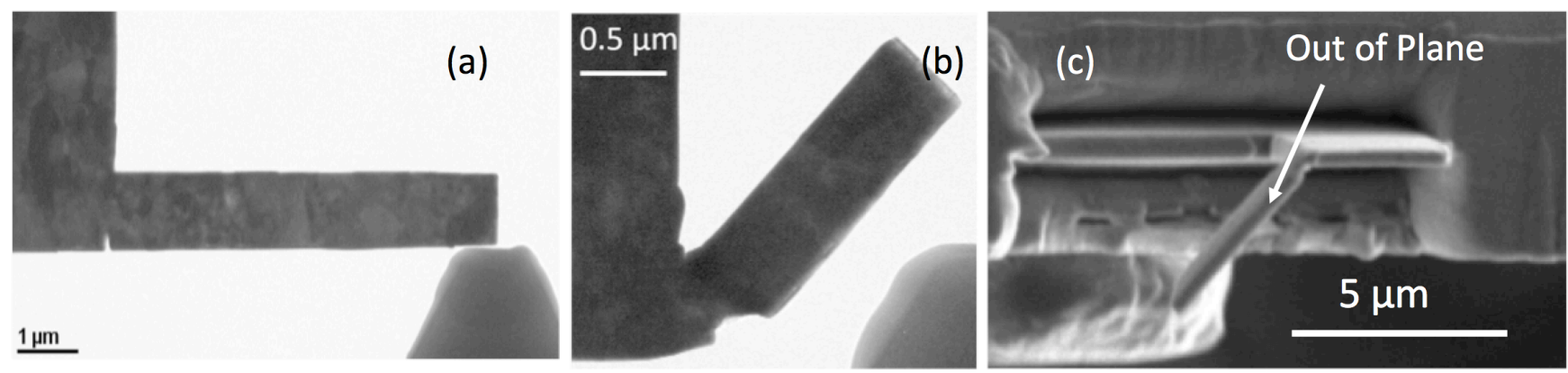

Figure 1. Still-frames from TEM videos of notched cantilever (a) before and (b) after bend testing; and (c) scanning electron microscopy image of cantilever bend out of plane after bend testing.

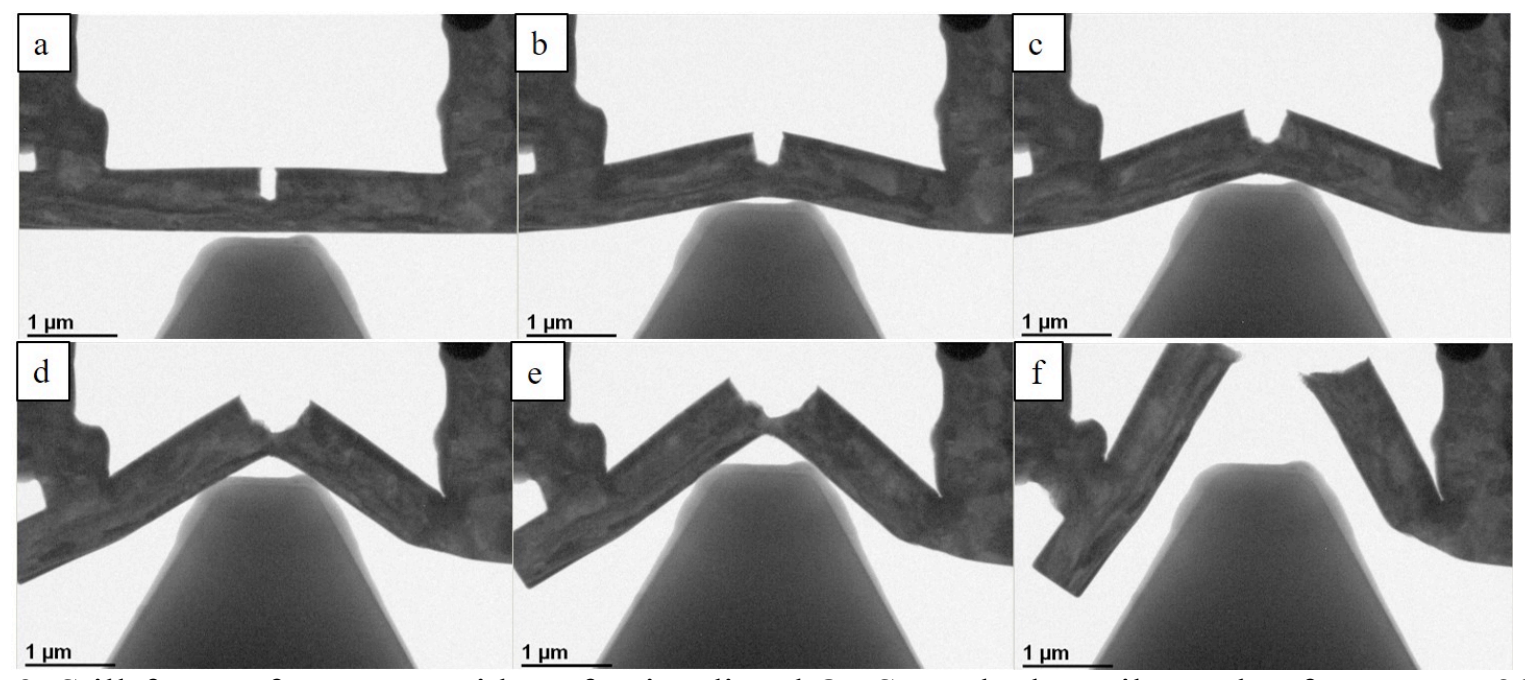

Figure 2. Still-frames from TEM video of unirradiated ODS notched cantilever that fractures at $202 \mu \mathrm{N}$. 